\title{
Performance Improvisation on 3D Converted 2D Unraveled Fingerprint
}

\author{
R.Anitha ${ }^{1}$, Dr.N.Sasirekha ${ }^{2}$ \\ ${ }^{I}$ PG Scholar, Biometrics and Cyber Security, Rathinam Technical Campus, Tamilnadu, India \\ ${ }^{2}$ Associate Professor \& Head, Information Technology, Rathinam College of Arts and Science, Tamilnadu, \\ India
}

\begin{abstract}
Fingerprint authentication system has been widely emerged in authentication purpose in public as well as in private organizations. Normally fingerprint image processing is done in form of acquiring 2-D images, by using Automatic Fingerprint Identification System. The captured $2 D$ texture will contain distortions. While comparing the enrolled distorted image from database with that of identification image, the results will be leads to false acceptance rate and false rejection rate. This paper deals with capturing of $3 D$ fingerprint image and processed to attain improvisation in the resultant fingerprint image. The proposed scan system is based on real-time capturing by using low $3 D$ sensor and with structured light illumination. This results in the generation of detailed in-depth ridge information and the texture of the finger in the form of $3 D$ image. Thus generated $3 D$ image is then converted to unraveled equal $2 D$ images by using the proposed best fit sphere algorithm. The NIST matching software is employed to analyze and evaluate the image quality. The comparison is made between the unraveled converted $2 D$ image and with its $2 D$ ink-rolled data. The NIST matching software is then applied for $2 D$ unraveled equivalent fingerprint images and results are analyzed. As a result, the fingerprints quality is improvised.
\end{abstract}

Key words: $2 D$ unraveled image, Best fit algorithm.

\section{Introduction}

Fingerprints are widely used for the identification and verification purpose of individuals such as in forensic, private/public concerns authentication systems, etc... There are six stages in the fingerprint identification system [1]: Image Acquisition, Image processing, Distinct Feature identification, Template creation and Template matching[10].

According to the Henry system, the patterns of fingerprints are classified into arch, whorl, and loop. Further the patterns break to tented arches or arches. Whorls are sub-divided into plain whorls, central loop whorls, double loop whorls and accidental whorls.

Now-a-days commonly used features are tented arch, arch, whorl, right loop, left loop. The ridge and minutiae types are crossover, core, bifurcation, ridge ending, island, delta and pore.

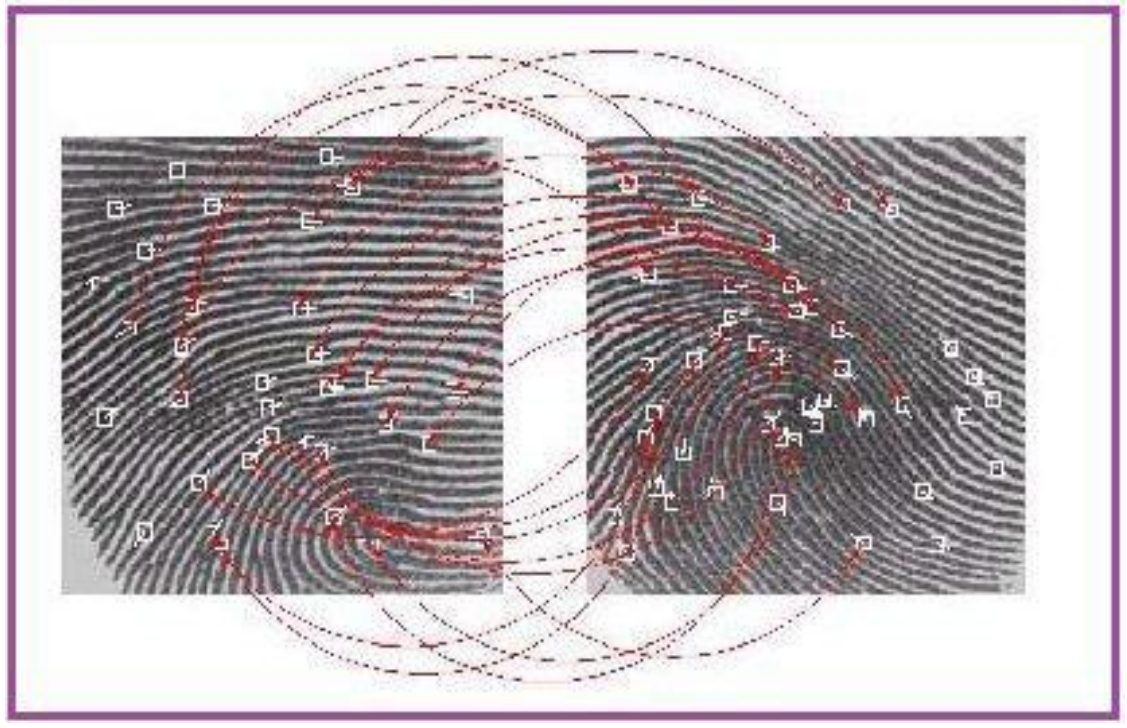

Fig 1 Matching fingerprints by using distinctive features

There are several technologies to acquire the fingerprint. The conventional technology is the optical 
technology in which a camera registers series of fingerprint images against the coated plastic or glass platen. Now-a-days silicon technology and ultrasound technology are used widely [1]. Sensors used for Image Acquisition are Optical Sensors, Capacitive Sensors, Pressure Sensors, Ultrasound Sensors, and Thermal Sensors.

Image acquisition and feature extraction are the most important stage in fingerprint processing. 2D images are prone to distortion while acquiring. Distorted images are not at all of high resolution. It will be a problem during verification process which is carried out from a database stored fingerprint image during enrollment process.

The 3D scan systems can able to scan finger and provides high resolution images[2]. And also the 3D scan is non contact with the finger. As 2D scans are solid scanners. The 3D scanned images do not contain any distortions. As a result, the compared and analyzed result will be accurate. False rejection rate will be minimized by using this method.

\section{Related Work}

The scan system used for fingerprint is multiple frequencies measuring profilometry technique based 3D scan system. To build this system, a non contact scan system was structured with high resolution[6], structured light illumination. Such that 3D scans can acquire all the five fingers and also the palm with better resolution as sufficient to have in-depth ridges level details. The system can be able to operate in both interfaces such as Autonomous Entry and also by Operator Controlled.

Acquired 3D fingerprint is applied in best fit spring algorithm for unraveling the 3D image to the 2D fingerprint. Some specialized software components are used which is structured by National Institute of Standards and Technology, for deriving the metrics for performance.

The comparison is made between the 2D finger-print images obtained by the conventional method[12] and 2D images made after unrolling 3D scans and acquired scans are quantified using the performance metrics. The results shown that both 2D inked and unraveled 3D image are similar in its distribution and trend[14].

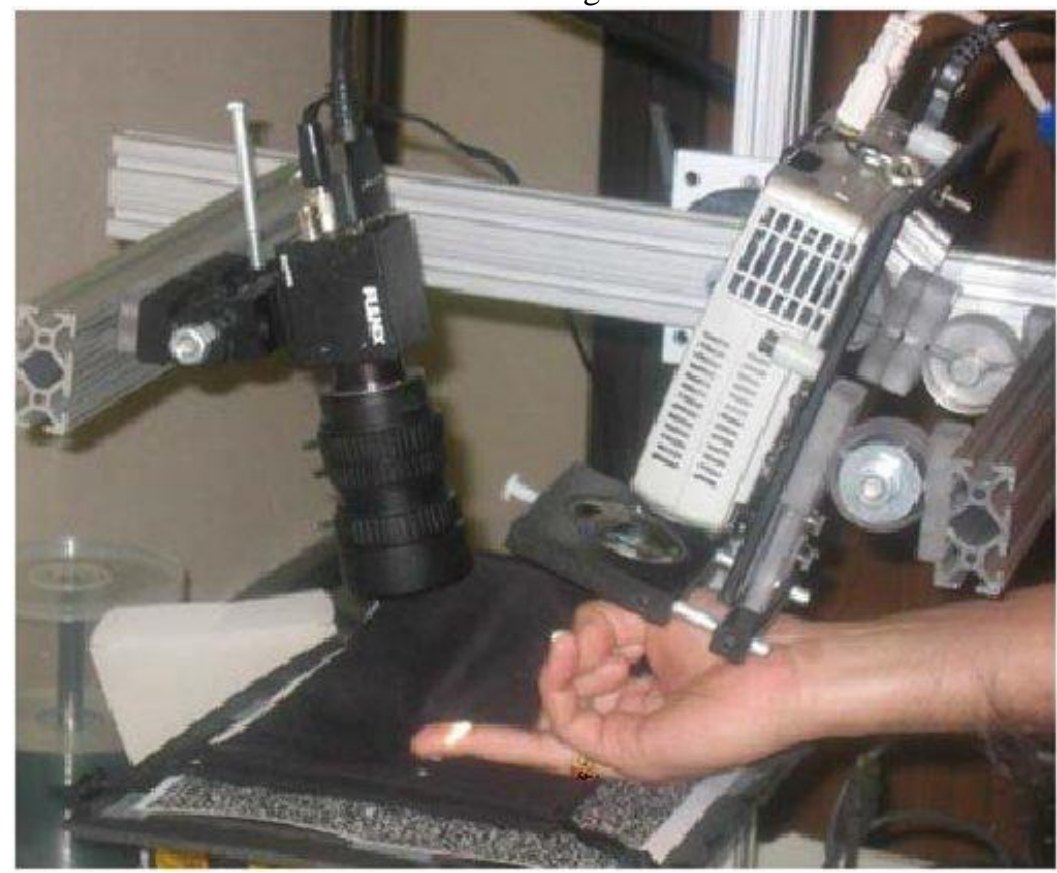

Fig 2 3D-Fingerprint scanner-Experimental Setup

\section{A. System Overview}

\section{Theoretical Methods And Implementation}

To assess the proposed finger-print $\mathrm{s}$ system, there is a need to find an $\mathrm{n}$ equivalent system to evaluate 3D fingerprint acquiring data and to make comparison with the 2D inked counterparts. Now-a-days most widely used fingerprint data are in 2D and indulged evaluation systems are also lies on 2D data[4]. From this criterion it is clearly understandable that proposed system can be employed after 3D fingerprint images are unraveled to $2 \mathrm{D}$ images and ridges extraction.

The 2D rolled equivalent data extracted from 3D fingerprint scanned data is essential for (a) using NIST software for minutiae extraction, (b) for comparing the results with store ink rolled fingerprint counterparts. 


\section{B. Calculation of sphere for best fit on 3D surface}

The foremost step in unraveling the fingerprint is for fitting 3Ddata in a sphere. The 3D surface of the sphere can be specified by its center point $\left(\mathrm{x}_{\mathrm{c}}, \mathrm{y}_{\mathrm{c}}, \mathrm{z}_{\mathrm{c}}\right)$ with the radius as "r". So, the main thing here is to generate a code to calculate the sphere's center point and also radius based on least squares, from which the sphere can minimizes sum of the squared distance from the sphere points to the corresponding fingerprint points.

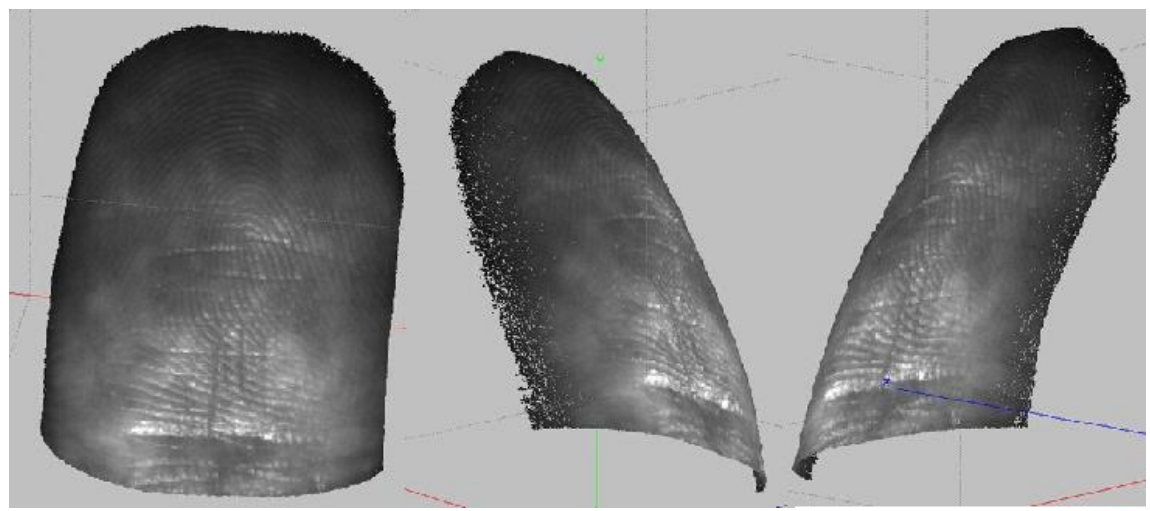

Fig.3 3different views of Scanned Fingerprint

The sum of the squared distance can be calculated as follows:

$$
\mathrm{d}_{0}=\left(\mathrm{x}_{\mathrm{f}}-\mathrm{x}_{\mathrm{c}}\right)^{2}+\left(\mathrm{y}_{\mathrm{f}}-\mathrm{y}_{\mathrm{c}}\right)^{2}+\left(\mathrm{z}_{\mathrm{f}}-\mathrm{z}_{\mathrm{c}}\right)^{2}
$$

Where $\left(\mathrm{x}_{\mathrm{f}} \mathrm{y}_{\mathrm{f}}, \mathrm{z}_{\mathrm{f}}\right)$ is the point from $3 \mathrm{D}$ fingerprint and $\left(\mathrm{x}_{\mathrm{c}}, \mathrm{y}_{\mathrm{c}}, \mathrm{z}_{\mathrm{c}}\right)$ is the sphere center. After computing the sum of squared distance. The distance from each point from surface of fingerprint to surface of sphere is given as:

$$
\mathrm{d}=\operatorname{sqrt}\left[\left(\mathrm{x}_{\mathrm{f}}-\mathrm{x}_{\mathrm{c}}\right)^{2}+\left(\mathrm{y}_{\mathrm{f}}-\mathrm{y}_{\mathrm{c}}\right)^{2}+\left(\mathrm{z}_{\mathrm{f}}-\mathrm{z}_{\mathrm{c}}\right)^{2}\right]-\mathrm{r}
$$

The parameter $\mathrm{s}$ is the scale value. We can solve the function by SVD decomposition of A, where A = UDVT and the last column of $\mathrm{V}$ is the solution. Thus, $\mathrm{a}=\mathrm{a} / \mathrm{s}, \mathrm{b}=\mathrm{b} / \mathrm{s}, \mathrm{c}=\mathrm{c} / \mathrm{s}$, and $\mathrm{d} 0=\mathrm{d} 0 / \mathrm{s}$. And $\mathrm{r}=$ $\operatorname{sqrt}((\mathrm{a} 2+\mathrm{b} 2+\mathrm{c} 2) / 4-\mathrm{d} 0)$, the center point $(\mathrm{xc}, \mathrm{yc}, \mathrm{zc})=(-\mathrm{a} / 2,-\mathrm{b} / 2,-\mathrm{c} / 2)$.

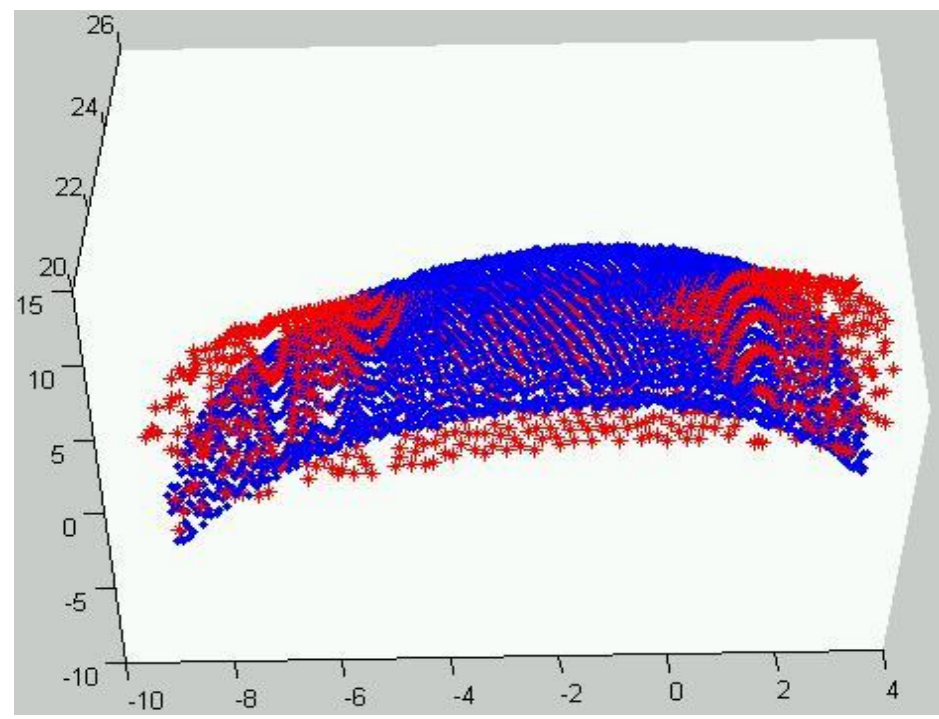

Fig.4 3D view of fingerprint and the sphere. Blue points represent points from the fingerprint data and the red points represent the best fit sphere.

The final step is to find the best fit sphere to change north axis of sphere to center of the finger-print. 


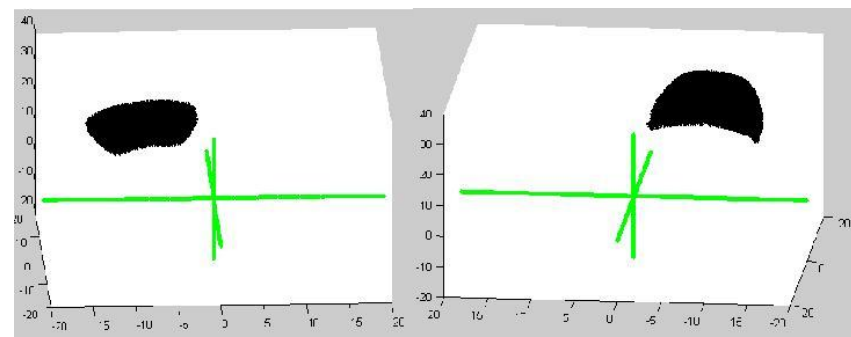

Fig. 4 shows that before the moving of the North Pole and the Unravel center is not on the fingerprint's surface.

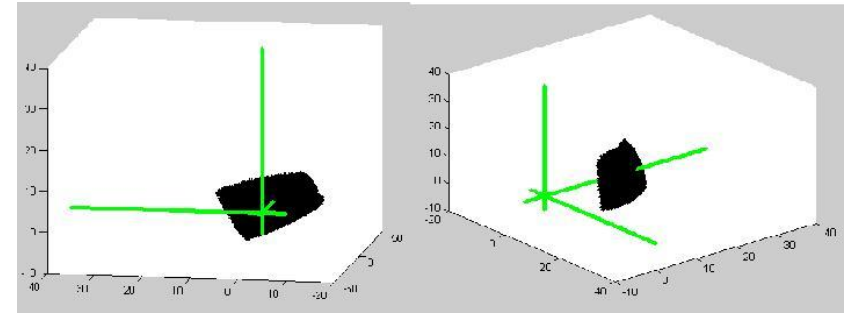

Fig.5 shows that after the rotation and translation, the unravel center is changed to the fingerprint's center.

\section{C.3D Fingerprint Unravel}

To unravel the 3D fingerprint data, first step is to create a grid. Commonly, the portion near to the camera is denser in points than the portion far from camera[14]. From this, it is very clear that fingerprint is not evenly distributed. So it is must to ensure that the points are evenly distributed on the surface.

To evaluate this, whole data set is converted from (x,y, z) to (theta, phi, rho) which is a sphere dimension. The uniform mesh is created which consists of phi and theta[13]. So that, if fingerprint is correctly fitted to sphere, then the created mesh will be projected onto fingerprint in equal manner and also value of every point on mesh is linearly computed based on fingerprint's data[7].

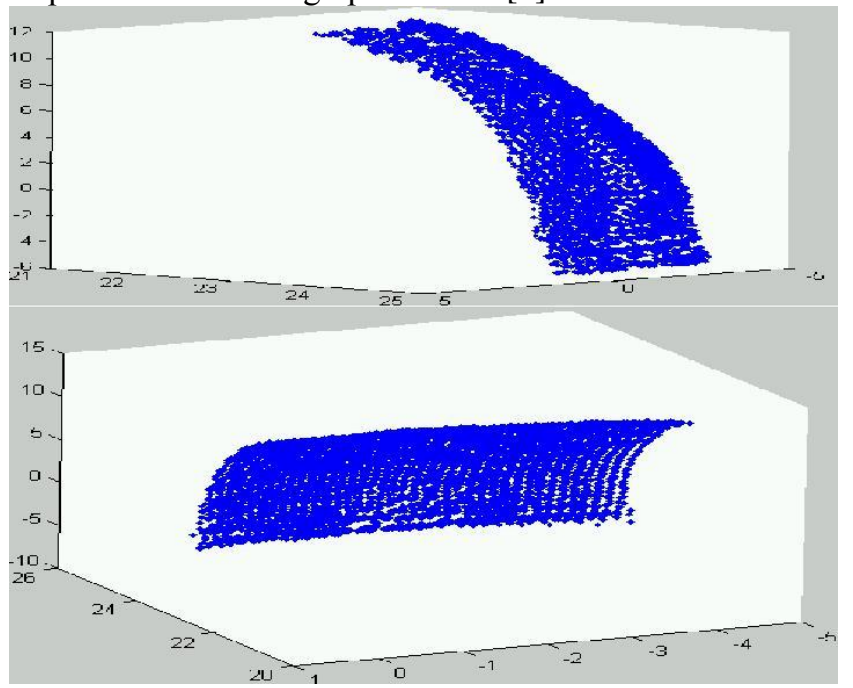

Fig.5 Different views of Grid which is created on 3D fingerprint.

The second step is to unravel the 3D surface[8]. The rho values of the fingerprint are integrated based on the theta and phi maps to create a new rho map of the fingerprint. This new rho value has mainly two uses[12]. First use is rho map is used to create the unraveled fingerprint. First use is rho map is used to correct the distortion of fingerprint caused by the algorithm of best fit sphere[5]. 


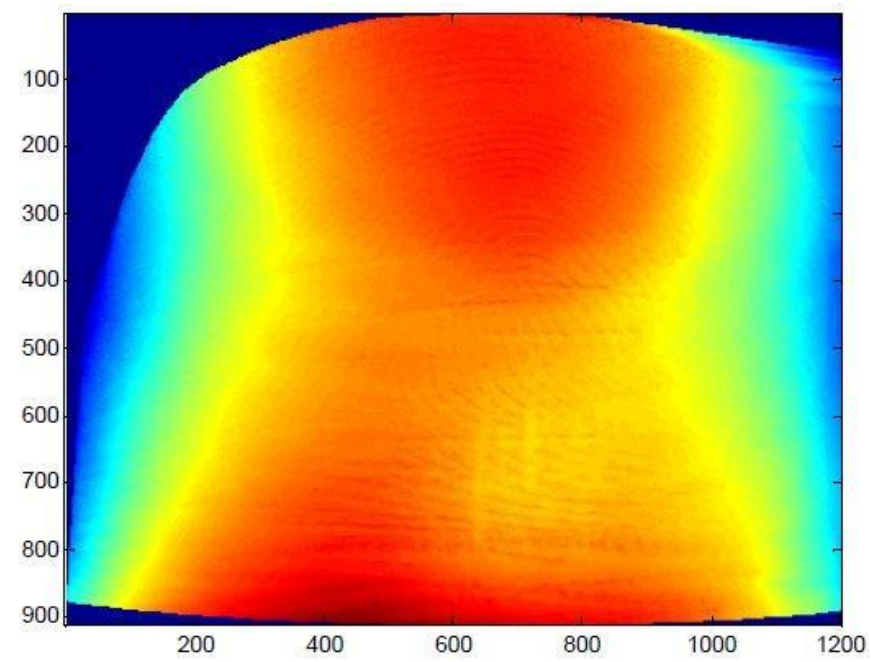

Fig.6 Unraveled 2D finger-print.

The third step is to apply filters to unravelled $2 \mathrm{D}$ fingerprint. To achieve this, a bound pass filter should be performed to abstract the finger ridges. Here, in order to apply a bound pass filter, we first perform a high pass filter and then subtract the high frequency.

However, there is lot of high frequency noises caused by the collecting of the 3D fingerprint process. The frequency of this noise is generally much higher than the frequency of the ridges on the finger. To reduce noise of the data an appropriate low pass filter is needed. After the filter, the un-meaningful filter created edge data is cropped out, and a hist-euqal function is performed to the data. The variance comparison is made before and after the distortion correction.

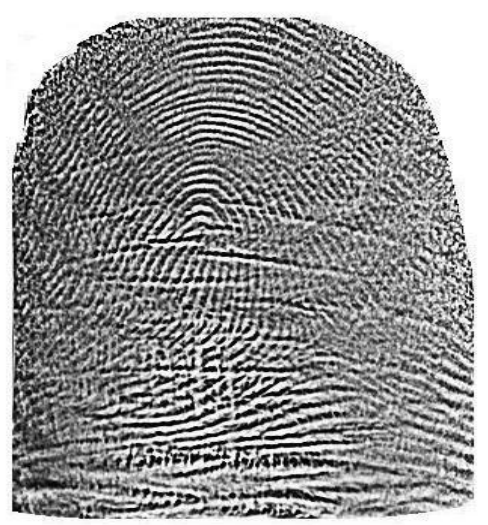

Fig.7 The 2D unraveled Fingerprint from 3D data.

\section{Applying NIST Software to Data}

The evaluation process is performed by NIST software. Here we will apply the binarization system (MINDTCT) and local quality system (NFIQ) to the 2D unraveled data to get the binary fingerprint and its corresponding quality map[9]. 


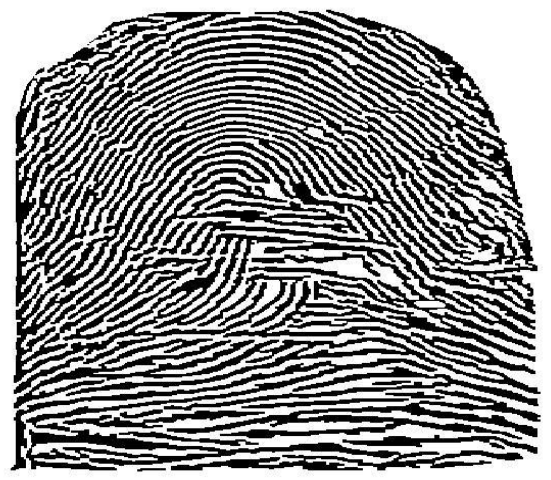

Fig. 8 Binarilized fingerprint of the 2D unraveled fingerprint from 3D.

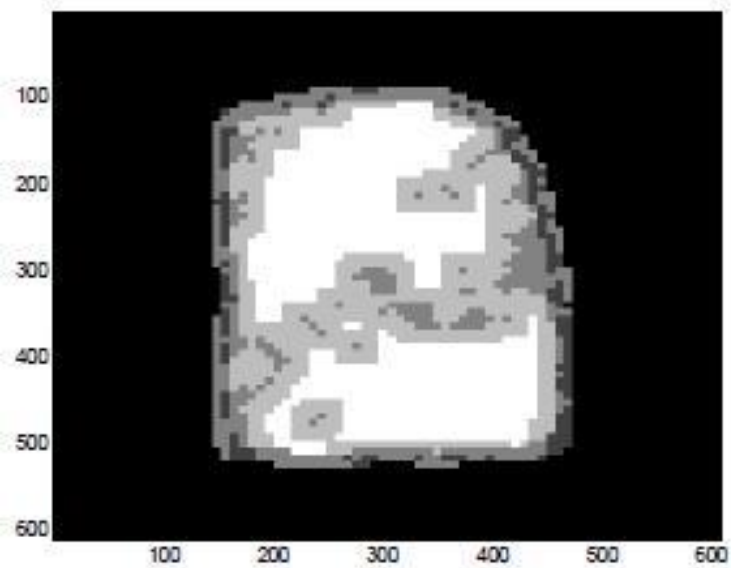

Fig. 9 Quality image of the 2D unraveled finger print. White color represents 4, which is the highest quality.

\section{E. 3D Unraveled Fingerprint Experimental Results}

The hypothesis on 2D fingerprint images generated after unraveling 3D fingerprint scans.

The $800 \times 800$ unraveled fingerprint images are run through the NIST PCASYS, MINDTCT, and NFIQ software systems generating the fingerprint class and the confidence number, minutiae classified into five different categories as $\min 05, \min 06, \min 075, \min 08, \min 09, \min 095$ and $\min 097$, and the number of blocks in quality zone 1, 2, 3, and 4 (qz1, qz2, qz3, qz4) along with an overall quality for the images. 


\begin{tabular}{|c|c|c|c|c|c|c|c|c|}
\hline \multirow[t]{2}{*}{ Subject } & \multirow[t]{2}{*}{ Hand } & \multirow[t]{2}{*}{ Digit } & \multicolumn{2}{|c|}{ PCASYS } & \multicolumn{2}{|c|}{ MINDTCT } & \multicolumn{2}{|l|}{ NFIQ } \\
\hline & & & Class & \begin{tabular}{|l} 
Conf. \\
No.
\end{tabular} & $\begin{array}{l}\text { Tol. } \\
\text { Min }\end{array}$ & $\begin{array}{l}\text { Rel| } \\
\text { Min }\end{array}$ & $\begin{array}{l}\text { Q. } \\
\text { Zone }\end{array}$ & Q. No. \\
\hline \multirow[t]{8}{*}{ Subject 0} & Left & D2 & W & 0.75 & 159 & 49 & 44.07 & 4 \\
\hline & & D3 & $R$ & 0.82 & 175 & 39 & 34.60 & 2 \\
\hline & & D4 & L & 1.00 & 159 & 42 & 39.62 & 3 \\
\hline & & D5 & L & 0.99 & 158 & 45 & 33.10 & 4 \\
\hline & Right & D2 & W & 0.90 & 139 & 49 & 46.74 & 3 \\
\hline & & D3 & $R$ & 0.99 & 145 & 50 & 54.24 & 3 \\
\hline & & D4 & R & 0.99 & 154 & 58 & 52.68 & 4 \\
\hline & & D5 & $R$ & 1.00 & 144 & 35 & 33.72 & 4 \\
\hline \multirow[t]{8}{*}{ Subject 1} & Left & D2 & L & 0.99 & 128 & 63 & 61.62 & 1 \\
\hline & & D3 & L & 1.00 & 148 & 63 & 53.35 & 2 \\
\hline & & D4 & W & 0.91 & 162 & 75 & 57.00 & 1 \\
\hline & & D5 & W & 0.84 & 149 & 82 & 64.67 & 1 \\
\hline & \begin{tabular}{|l|} 
Right \\
\end{tabular} & D2 & W & 0.97 & 117 & 56 & 54.22 & 1 \\
\hline & & D3 & W & 0.71 & 130 & 59 & 57.55 & 1 \\
\hline & & D4 & W & 0.69 & 171 & 66 & 55.17 & 1 \\
\hline & & D5 & W & 0.65 & 157 & 57 & 46.50 & 2 \\
\hline \multirow[t]{8}{*}{ Subject 2} & Left & D2 & R & 0.59 & 254 & 33 & 13.59 & 1 \\
\hline & & D3 & L & 0.95 & 268 & 18 & 10.42 & 3 \\
\hline & & D4 & L & 0.65 & 216 & 19 & 10.86 & 5 \\
\hline & & D5 & L & 0.82 & 150 & 27 & 19.66 & 4 \\
\hline & \begin{tabular}{|l|} 
Right \\
\end{tabular} & $\mathrm{D} 2$ & R & 0.34 & 261 & 27 & 15.09 & 3 \\
\hline & & D3 & L & 0.66 & 200 & 3 & 190 & 4 \\
\hline & & D4 & W & 0.67 & 256 & 20 & 11.18 & 5 \\
\hline & & D5 & $R$ & 0.54 & 211 & 9 & 7.74 & 4 \\
\hline
\end{tabular}

Table 1 Results of running PCASYS, MINDTCT and NFIQ.

\section{Unraveled Fingerprint}

\section{Comparison Between 2d Inked And 3d}

After evaluating the 3D fingerprint scans, we will compare the 2D rolled inked fingerprints and the 3D unraveled fingerprint images where the unraveling program is best fit sphere. We will use the parameters: number of foreground blocks in quality zone 4 (qz4), number of minutiae with quality greater than 0.75 (min075), and the confidence number.

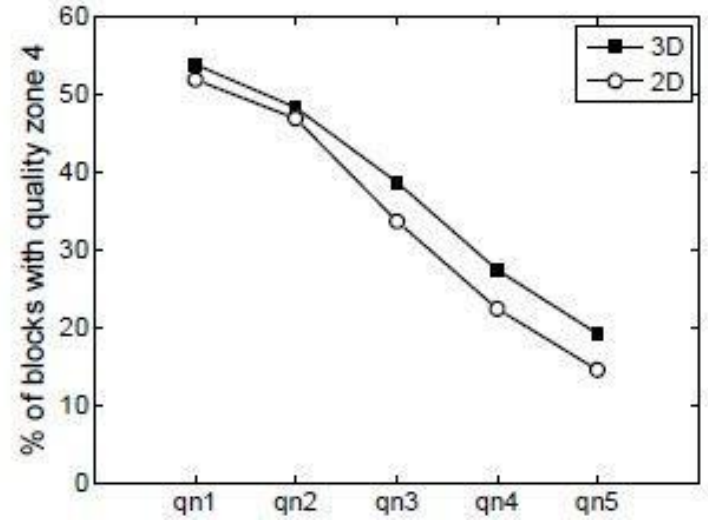

Fig. 9 Overall Quality Number

The Fig. 9 shows that the fingerprints from 3D unraveled have better performance in the percentage of blocks with quality number 4 . The figure also shows the variation of qz4 blocks with the quality number for both 2D and 3D fingerprints. Statistical analysis shows a significant difference in the values between 2D images and 3D images for qn3, qn4, and qn5, whereas the values for qn1 and qn2 are comparable. However, both the 
groups followed similar trend of decrease in the number of quality zone 4 blocks with decrease in the quality from best to unusable.

\section{Conclusion}

This paper presents a new approach for 3-D fingerprint acquisition and matching using structured light illumination and 2-D equivalent 3-D image data processing. Both the processes of acquiring the 3D fingerprint and post processing the data are free of distortion. We employed software systems developed by the National Institute of Standards and Technology (NIST), used for conventional 2-D fingerprints, to evaluate the performance of 3-D fingerprints after unraveling them into 2-D rolled equivalent images. Compared with the 2D rolled inked counterpart, the new 3-D approach provides competitive performance with more user convenience, higher robustness to experimental conditions, faster data collection speed, and free of distortion. And also we show that higher the quality of the fingerprint is, higher possibility of better matching performance the fingerprint will achieve.

\section{References}

[1] Veeraganesh Yalla. Optimal Phase Measuring Profilometry Techniques for Static and Dynamic 3D Data Acquisition. PhD Dissertation, University of Kentucky, Lexington, USA. July, 2006.

[2] Abhishika Fatehpuria. Performance Evaluation of Non-contact 3D Fingerprint Scanner. MS Thesis, University of Kentucky, Lexington, USA. November, 2006

[3] Veeraganesh Yalla. Multi-frequency Phase Measuring Profilomety. MS Thesis, University of Kentucky, Lexington, USA. December 2004.

[4] C. Guan. Composite Pattern for Single Frame 3D Acquisition. PhD Dissertation, University of Kentucky, Lexington, USA. December 2004.

[5] C Guan, L. G. Hassebrook and D. L. Lau. "Composite Structure Light Pattern for Three Dimensional Video”. Opt. Exp., 11, 406417, 2003.

[6] International Biometrics Group. Biometrics Market and Industry Report 2006-2010, Jan 2006.

[7] D. Maltoni, D. Maio, A. K. Jain, and S. Prabhakar. Handbook of Fingerprint Recognition. Sprinter-Verlag, 2003.

[8] http://en.wikipedia.org/wiki/Fingerprint

[9] Olsen, Robert D., Sr. (1972) “The Chemical Composition of Palmar Sweat” Fingerprint and Identification Magazine Vol 53(10).

[10] Tewari RK, Ravikumar KV. History and development of forensic science in India. J. Postgrad Med 2000,46:303-308.

[11] The History of Fingerprints. http://onin.com/fp/fphistory.html.

[12] A. K. Jain, R. Bolle, and S. Pankanti, editors. Biometrics-Personal Identification in Networked Society. Kluwer Academic Publishers. 1999.

[13] H. C. Lee and R. Gaensslen, editors. Advances in Fingerprint Technology. Florida: CRC Press, 2 Edition, 2001.

[14] B. Laufer. History of Fingerprint System. Washington: Government Printing Office, 1912.

[15] F. Galton. Fingerprints. McMillan, London, 1892 